\title{
Systematic Evaluation of Exertional Hyperthermia in Children and Adolescents With Hypohidrotic Ectodermal Dysplasia: An Observational Study
}

\author{
JOHANNA E. HAMMERSEN, VALENTIN NEUKAM, KAI-DIETRICH NÜSKEN, AND HOLM SCHNEIDER \\ Department of Pediatrics, German Competence Centre for Children with Ectodermal Dysplasias, University of Erlangen-Nürnberg, \\ 91054 Erlangen, Germany
}

\begin{abstract}
To evaluate exertional overheating and the impact of physical exercise on individuals with hypohidrotic ectodermal dysplasia (HED) and to assess protective effects of cooling devices, 13 boys and male adolescents with X-linked HED (XLHED) and agematched healthy male controls were studied during standardized exercise on a bicycle ergometer at ambient temperatures of 25 and $30^{\circ} \mathrm{C}$, without cooling and with evaporative skin cooling devices at $30^{\circ} \mathrm{C}$. Body core temperature during and after exercise, heart rate, performance, endurance, and serum lactate were investigated. XLHED subjects experienced a significantly greater rise in body temperature after cycling than healthy controls, and their body temperature remained elevated longer. Maximum heart rates and lactate values did not differ significantly between XLHED and control groups. Application of skin cooling devices led to a clinically relevant attenuation of exertional hyperthermia in XLHED patients, and a previous tendency toward lower performance disappeared. This first systematic study of the effects of physical exercise on HED patients demonstrates a rapid and lasting body temperature increase in XLHED subjects after cycling, posing them at risk of exerciseinduced hyperthermia. External evaporative skin cooling attenuates exertional overheating in HED patients and may facilitate their participation in athletic activities and professional life. (Pediatr Res 70: 297-301, 2011)
\end{abstract}

$\mathrm{H}$ ypohidrotic ectodermal dysplasia (HED) is a complex genetic disorder characterized by hypoplasia of sweat, sebaceous, submucous, meibomian, and mammary glands, sparse hair, and missing or malformed teeth (1). Most frequently this disease is caused by an X-linked genetic defect of the epithelial morphogen ectodysplasin $\mathrm{A}$, a member of the TNF superfamily. Mutations in the genes encoding components of the ectodysplasin A signaling pathway can lead to autosomal forms of HED (2). Children affected by HED have dry and wrinkled skin and suffer significant morbidity, including disturbed thermoregulation, recurrent infections of the respiratory tract, and failure to thrive $(1,2)$. The greatest risk arises from their inability to sweat resulting in episodes of

Received November 10, 2010; accepted March 25, 2011.

Correspondence: Holm Schneider, M.D., Department of Pediatrics, University of Erlangen-Nürnberg, Loschgestr. 15, 91054 Erlangen Germany; e-mail: holm.schneider@uk-erlangen.de

Supported by the German-Swiss-Austrian ectodermal dysplasia patient support group and by a grant from Pervormance GmbH (Ulm, Germany).

This study is registered in the national registry of clinical trials (No. NCT01135888) at ClinicalTrials.gov.

J.E.H. and V.N. contributed equally to this study. severe hyperthermia. Mortality of HED is highest during the first year of life, but remains elevated throughout early childhood (2-4). Later in life, ambient temperatures of more than $25^{\circ} \mathrm{C}$, exposure to the sun, and physical exercise may cause dangerous hyperthermia, often constraining HED patients in their professional careers and daily activities, especially in sports and traveling. Body core temperatures increase during exercise also in healthy individuals, which may accelerate neuromuscular fatigue, reduce endurance, and performance $(5,6)$, or may even lead to dangerous conditions such as exertional heat stroke (7).

Exertional hyperthermia in individuals with X-linked HED (XLHED) was already reflected in the work of Charles Darwin, who had received correspondence from India depicting a family in which 10 men had sparse hair, abnormal teeth, and unexpected dryness of the skin during hot weather. Absence of sweating was understood as a hazard when working out in the fields (8). The affected men may have survived the heat only by tipping buckets of water over each other. However, exertional hyperthermia in HED has not yet been investigated in a systematic manner. To study the effects of physical exercise on pediatric HED patients and to determine levels of activity they tolerate and may engage in without health hazards, we exposed boys and male adolescents with XLHED together with age-matched controls to standardized physical exercise on a bicycle ergometer. We investigated their body core temperature during and after exertion, heart rate, performance, and serum lactate and evaluated the application of cooling devices in this setting.

\section{SUBJECTS AND METHODS}

Study design. This is an observational study of individuals with XLHED and age-matched healthy male volunteers as controls.

Subjects. Thirteen male XLHED patients seen at the Competence Centre for Children with Ectodermal Dysplasias of the University Hospital Erlangen participated in the study (registered at clinicaltrials.gov NCT01135888). Controls were 15 age-matched healthy male volunteers. Enrollment of the subjects was between May 2009 and February 2010. All subjects and their parents/guardians gave written informed consent to participate.

Criteria for exclusion were acute febrile illness, acute or chronic heart disease, arterial hypertension, gastrointestinal diseases, implantable electronic devices, MRI investigation scheduled for the $5 \mathrm{~d}$ subsequent to the study, and clinical signs or diagnostic findings of dehydration. To exclude hypohydra-

Abbreviations: HED, hypohidrotic ectodermal dysplasia; XLHED, X-linked hypohidrotic ectodermal dysplasia 
tion, body composition was evaluated by body impedance analysis (BIA 101; Medi Cal HealthCare, Germany) before bicycle ergometry. The parameters determined were total body water relative to body weight, extracellular water relative to total body water and body cell mass, and fat mass relative to body weight. The study was approved by the ethics committee of the University of Erlangen-Nürnberg and conducted in compliance with German legal requirements.

Main outcome measures. The main outcome measures were body core temperature during and after exercise, heart rate, endurance, performance, and serum lactate. Body core temperature was measured immediately before and $4,8,12,16,20,24,30,40,60$, and 90 min after initiation of exercise using an ingestible thermometer pill (CorTemp; HQ, Inc., Palmetto). These thermometer pills with a crystal sensor and a silver oxide battery in a silicone capsule (one time usage, diameter of $11 \mathrm{~mm}$ ) were swallowed with some water. External recording of body core temperature was started $\sim 30$ min after ingestion of a calibrated pill, which is a reliable means of transmitting body core temperature data continuously for at least $8 \mathrm{~h}$ and provides more accurate values than traditional ear measurements. Basal values were noted when no changes of body temperature could be observed anymore, and the subject was then told to start cycling. As maximum values of body temperature were recorded at 30 or $40 \mathrm{~min}$ after initiation of exercise in all cases, only the time points of $0,30,40,60$, and 90 min were used for further analysis. Heart rate was monitored continuously. Physical strain was $0.5 \mathrm{~W} / \mathrm{kg}$ body weight at the beginning and was increased every $4 \mathrm{~min}$ by $0.5 \mathrm{~W} / \mathrm{kg}$ body weight. Serum lactate was determined within $2 \mathrm{~min}$ after discontinuation of cycling.

Study setting. Six XLHED children, six XLHED adolescents, six healthy children, and six healthy adolescents performed bicycle ergometry at an ambient temperature of $25^{\circ} \mathrm{C}$ for a maximum of $30 \mathrm{~min}$. Abort criteria were a body core temperature of more than $40^{\circ} \mathrm{C}$, an increase in heart rate above an age-adjusted threshold, or subjective exhaustion. In all cases, exercise was continued until subjective exhaustion.

In a second trial, five adolescents with XLHED and five healthy male adolescents cycled three times at an ambient temperature of $30^{\circ} \mathrm{C}$ and relative humidity of $40 \%$ for a maximum of $30 \mathrm{~min}$ : once without any cooling device, once with a Cooline evaporative fleece cooling vest (Pervormance $\mathrm{GmbH}$, Ulm, Germany), and once wearing a cooling vest and a cooling bandana (Pervormance $\mathrm{GmbH}$ ). Subjects started either without cooling or with the cooling vest, being attributed randomly to one of these groups, and repeated the exercise after recreation for at least $4 \mathrm{~h}$. The third ergometry was performed on a different day.

Statistical analyses. All data showed normal distribution and are displayed as mean $\pm \mathrm{SD}$. Bivariate analyses were performed using $t$ tests. Courses of body temperature were compared using ANOVA. Because of multiple comparisons, an adjusted $p$ value (two-tailed) of $<0.01$ was considered significant in all analyses.

\section{RESULTS}

First, we determined differences in the course of body temperature and performance between HED patients and control individuals during exercise at an ambient temperature of $25^{\circ} \mathrm{C}$. Six boys aged between 7 and $12 \mathrm{y}$ with XLHED and six male adolescents aged between 14 and 18 y were studied during bicycle ergometry and compared with age-matched healthy male volunteers. Characteristics of the subjects are depicted in Table 1. There were no significant differences in body weight, height, or hours of weekly recreational exercise between XLHED and control groups. None of the participants showed signs of dehydration (as measured by BIACORE assays) or acute febrile illness.

All subjects underwent bicycle ergometry with increasing strain until subjective exhaustion. At an ambient temperature of $25^{\circ} \mathrm{C}$, children cycled for $19.3 \pm 4.0 \mathrm{~min}$ on average, whereas adolescents aborted after a mean of $23.0 \pm 2.9 \mathrm{~min}$. At this point, mean performance was $2.63 \pm 0.48 \mathrm{~W} / \mathrm{kg}$ in children and $3.00 \pm 0.43 \mathrm{~W} / \mathrm{kg}$ in adolescents. Endurance and

Table 1.Characteristics of XLHED, control subjects, and performance during bicycle ergometry at $25^{\circ} \mathrm{C}$

\begin{tabular}{|c|c|c|c|c|c|c|c|c|c|c|}
\hline Subject & $\begin{array}{l}\text { Age } \\
(\mathrm{y})\end{array}$ & $\begin{array}{l}\text { Weight } \\
\text { (kg) }\end{array}$ & $\begin{array}{l}\text { Height } \\
(\mathrm{cm})\end{array}$ & $\begin{array}{c}\text { Total body } \\
\text { fat }(\%)\end{array}$ & $\begin{array}{c}\text { Recreational } \\
\text { exercise (h/wk) }\end{array}$ & $\begin{array}{l}\text { Basal body core } \\
\text { temperature }\left({ }^{\circ} \mathrm{C}\right)\end{array}$ & $\begin{array}{c}\text { Performance } \\
(\mathrm{W} / \mathrm{kg})\end{array}$ & $\begin{array}{l}\text { Endurance } \\
\text { (min) }\end{array}$ & $\begin{array}{l}\text { Maximal heart } \\
\text { rate }(/ \mathrm{min})\end{array}$ & $\begin{array}{c}\text { Lactate } \\
(\mathrm{mmol} / \mathrm{L})\end{array}$ \\
\hline \multicolumn{11}{|l|}{ Control children } \\
\hline C-Ch1 & 12 & 46.0 & 160 & 11.9 & 4.0 & 37.4 & 2.50 & 20 & 202 & 6.9 \\
\hline C-Ch2 & 11 & 32.0 & 145 & 11.4 & 3.0 & 37.3 & 3.00 & 24 & 181 & 4.7 \\
\hline C-Ch3 & 11 & 34.0 & 143 & 20.3 & 2.5 & 37.0 & 2.50 & 18 & 204 & 2.5 \\
\hline C-Ch4 & 10 & 54.7 & 157 & 26.8 & 1.0 & 36.9 & 2.00 & 13 & 179 & 2.6 \\
\hline C-Ch5 & 9 & 32.0 & 139 & 18.4 & 2.5 & 37.4 & 3.00 & 21 & 176 & 4.3 \\
\hline C-Ch6 & 8 & 30.3 & 133 & 12.7 & 2.5 & 36.9 & 2.50 & 20 & 168 & 5.6 \\
\hline \multicolumn{11}{|c|}{ XLHED children } \\
\hline ED-Ch1 & 12 & 39.5 & 156 & 13.1 & 2.5 & 36.7 & 2.50 & 17 & 179 & 2.8 \\
\hline ED-Ch2 & 11 & 32.0 & 150 & 12.9 & 4.0 & 37.0 & 3.50 & 26 & 198 & 7.9 \\
\hline ED-Ch3 & 11 & 50.0 & 165 & 9.5 & 2.5 & 37.2 & 3.00 & 22 & 186 & 11.9 \\
\hline ED-Ch4 & 10 & 34.0 & 157 & 9.8 & 2.5 & 36.3 & 2.00 & 13 & 164 & n.d. \\
\hline ED-Ch5 & 9 & 38.9 & 143 & n.d. & 2.5 & 36.8 & 3.00 & 21 & 173 & 4.2 \\
\hline ED-Ch6 & 8 & 26.5 & 137 & 1.9 & 2.5 & 36.8 & 2.00 & 16 & 177 & 4.1 \\
\hline \multicolumn{11}{|c|}{ Control adolescents } \\
\hline C-Ad1 & 18 & 74.0 & 182 & 17.4 & 2.5 & 36.8 & 3.50 & 26 & 196 & 3.5 \\
\hline C-Ad2 & 16 & 64.8 & 164 & 16.5 & 4.0 & 37.4 & 3.00 & 24 & 188 & 2.7 \\
\hline C-Ad3 & 15 & 53.5 & 169 & 14.6 & 2.5 & 37.0 & 2.50 & 20 & 167 & 2.5 \\
\hline C-Ad4 & 15 & 68.0 & 179 & 20.4 & 1.0 & 37.3 & 3.00 & 24 & 193 & 3.7 \\
\hline C-Ad5 & 15 & 58.0 & 174 & 17.3 & 2.5 & 37.2 & 3.50 & 26 & 200 & 4.7 \\
\hline C-Ad6 & 14 & 60.3 & 172 & 10.9 & 3.0 & 37.0 & 3.00 & 24 & 185 & 4.6 \\
\hline \multicolumn{11}{|c|}{ XLHED adolescents } \\
\hline ED-Ad1 & 18 & 70.0 & 182 & 12.4 & 1.0 & 36.4 & 3.00 & 21 & 189 & 6.9 \\
\hline ED-Ad2 & 16 & 61.0 & 183 & 15.2 & 2.5 & 36.8 & 3.00 & 24 & 195 & 6.3 \\
\hline ED-Ad3 & 15 & 80.0 & 174 & 28.8 & 2.5 & 37.0 & 2.00 & 16 & 206 & 10.6 \\
\hline ED-Ad4 & 15 & 61.0 & 183 & 18.8 & 4.0 & 36.9 & 3.50 & 25 & 201 & 5.3 \\
\hline ED-Ad5 & 15 & 50.0 & 171 & 16.8 & 2.5 & 36.9 & 3.00 & 24 & 196 & 6.5 \\
\hline ED-Ad6 & 14 & 38.9 & 166 & 2.5 & 2.5 & 36.9 & 3.00 & 22 & 199 & 22.6 \\
\hline
\end{tabular}

n.d., not determined. 
performance of the two XLHED groups did not differ significantly from those of the control groups.

As expected, differences in the course of body temperature during and after exercise between the XLHED subjects and the controls were observed (children: $p<0.0002$; adolescents: $p=0.0003$ ). Maximum body temperature was measured either at 30 or at $40 \mathrm{~min}$ after initiation of exercise and declined thereafter. XLHED children and adolescents showed a significantly greater rise of body temperature during workout than the control groups, and their body temperature remained elevated longer than in healthy subjects (Fig. 1). In children suffering from XLHED, body temperature rose by $1.54 \pm 0.66^{\circ} \mathrm{C}$, reaching $38.4 \pm 0.7^{\circ} \mathrm{C}$ at $30 \mathrm{~min}$. In XLHED adolescents, maximum body temperature was $38.8 \pm 0.5^{\circ} \mathrm{C}$ after an increase of $1.93 \pm 0.34^{\circ} \mathrm{C}$. Body temperature difference at the 1-h time point was still significantly higher in HED males of both age groups than in controls (Fig. 1; children: $p=0.0097$; adolescents: $p<0.0001$ ). Thus, XLHED subjects experienced a rapid, potentially endangering increase in body temperature in conjunction with bicycle ergometry even under normal ambient temperature.

To evaluate the physical strain of exercise, maximum heart rate during ergometry was documented, and serum lactate as a marker of metabolic stress was measured (Table 1). In subject ED-Ad6 who showed a very high lactate value together with a low body fat content, an additional inborn error
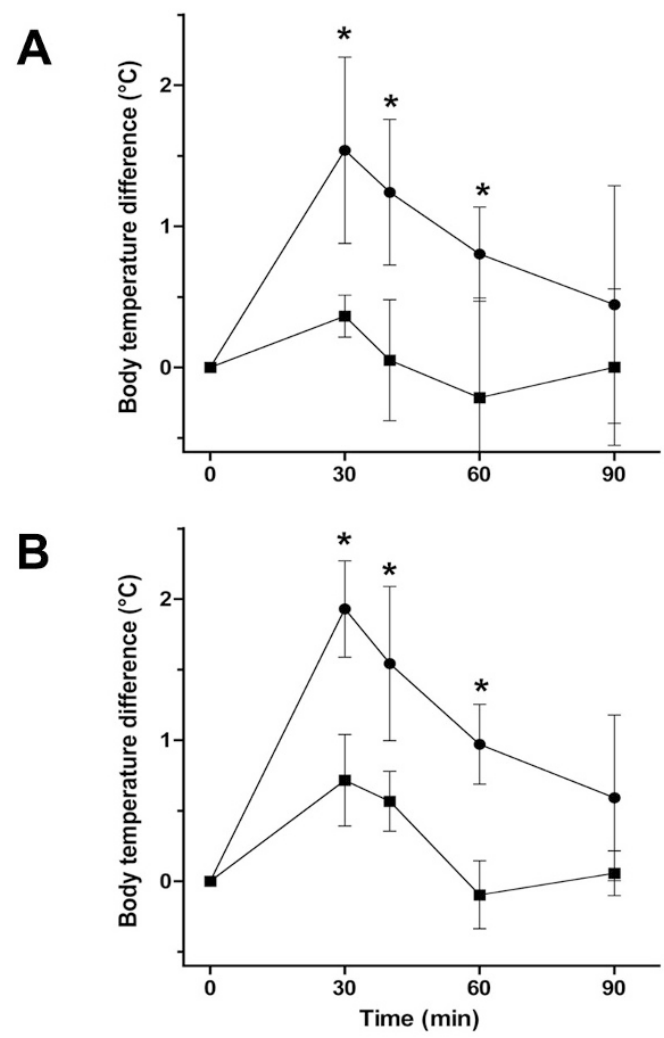

Figure 1. Exercise-induced changes of body core temperature in children $(A)$ and adolescents $(B)$ at an ambient temperature of $25^{\circ} \mathrm{C}$. Subjects cycled for a maximum of $30 \mathrm{~min}$. Data are displayed as mean \pm SD. XLHED subjects are displayed as circles and control subjects as squares. * indicates significant differences between XLHED and control subjects. of metabolism had been excluded before this study. There was no significant difference in maximum heart rates or lactate values between XLHED and control groups.

Second, XLHED patients and control individuals were exposed to physical exercise under conditions resembling a hot summer day with an ambient temperature of $30^{\circ} \mathrm{C}$ and a relative humidity of $40 \%$. In this setting, the benefit of cooling devices for adolescents with XLHED was also evaluated. Five male individuals with XLHED aged 12-18 y and 5 agematched healthy male controls underwent bicycle ergometry at an ambient temperature of $30^{\circ} \mathrm{C}$ without cooling device, with a cooling vest, or with a cooling vest plus a cooling bandana.

XLHED and control subjects did not differ significantly in weight, size, or body temperature before exercise (Table 2). Without cooling device, XLHED subjects discontinued ergometry after $19.0 \pm 2.4 \mathrm{~min}$, whereas healthy males cycled for $23.4 \pm 2.7 \mathrm{~min}$. Performance was $2.5 \pm 0.4 \mathrm{~W} / \mathrm{kg}$ in XLHED adolescents without cooling device and $3.2 \pm 0.4$ $\mathrm{W} / \mathrm{kg}$ in the control group. These trends toward shorter selfchosen duration of exercise $(p=0.027)$ and lower performance $(p=0.025)$ in XLHED adolescents in comparison with control adolescents at an ambient temperature of $30^{\circ} \mathrm{C}$ were not surprising. Similar to an ambient temperature of $25^{\circ} \mathrm{C}$, the course of body temperature after exercise differed significantly between XLHED subjects and controls $(p<0.0001)$. Body temperature rose to a maximum of $38.1 \pm 0.4^{\circ} \mathrm{C}$ after $30 \mathrm{~min}$ in controls but reached a maximum of $39.3 \pm 0.5^{\circ} \mathrm{C}$ in XLHED individuals. It also remained elevated longer in XLHED individuals than in healthy age-matched males (Fig. 2). There were no significant differences in lactate and maximum heart rate between the two groups.

In XLHED patients, cooling had a "dose-dependent" effect on body temperature during and after exercise. XLHED adolescents who wore a cooling vest showed a tendency toward lower body temperature compared with ergometry without cooling ( $p=0.0252$; Fig. 2). When these subjects wore both a cooling vest and a cooling bandana, the course of body temperature after cycling differed significantly from that registered after ergometry without cooling ( $p=0.0009$; Fig. 2). The maximum of body temperature at $30 \mathrm{~min}$ was $38.5 \pm 0.2^{\circ}$ when XLHED patients were equipped with both cooling devices, in contrast to $39.3 \pm 0.5^{\circ} \mathrm{C}$ without cooling $(p=$ 0.0071 ), and the highest individual temperature measured was $38.7^{\circ} \mathrm{C}$ compared with $39.6^{\circ} \mathrm{C}$ without cooling. Thus, body temperature increased significantly less in XLHED patients when these were wearing a cooling vest together with a cooling bandana, and normal body temperature was reached sooner (Fig. 2).

Moreover, a combination of cooling vest and cooling bandana in XLHED adolescents led to a slightly increased performance index $(p=0.013)$. Wearing a cooling vest only or wearing both cooling devices had neither significant influence on endurance nor on stress parameters in XLHED individuals (Table 2). However, when XLHED subjects equipped with both cooling devices were compared with uncooled control adolescents, the tendency toward lower performance and shorter self-chosen duration of exercise in XLHED adoles- 
Table 2. Performance of XLHED and control subjects during bicycle ergometry at $30^{\circ} \mathrm{C}$

\begin{tabular}{|c|c|c|c|c|c|c|c|c|c|c|c|c|c|c|c|}
\hline \multirow[b]{2}{*}{ Subject } & \multirow{2}{*}{$\begin{array}{l}\text { Age } \\
(y)\end{array}$} & \multirow{2}{*}{$\begin{array}{c}\text { Weight } \\
(\mathrm{kg})\end{array}$} & \multirow{2}{*}{$\begin{array}{l}\text { Height } \\
(\mathrm{cm})\end{array}$} & \multicolumn{3}{|c|}{$\begin{array}{l}\text { Basal body core } \\
\text { temperature }\left({ }^{\circ} \mathrm{C}\right)\end{array}$} & \multicolumn{3}{|c|}{ Performance (W/kg) } & \multicolumn{3}{|c|}{ Endurance (min) } & \multicolumn{3}{|c|}{ Lactate $(\mathrm{mmol} / \mathrm{L})$} \\
\hline & & & & $\varnothing$ & $\mathrm{V}$ & $v+b$ & $\varnothing$ & v & $v+b$ & $\varnothing$ & $\mathrm{V}$ & $v+b$ & $\varnothing$ & V & $v+b$ \\
\hline C30-1 & 18 & 81.5 & 181 & 37.4 & 37.4 & 37.0 & 3.5 & 3.0 & 3.5 & 25.0 & 24.0 & 25.0 & 6.3 & 7.1 & 5.4 \\
\hline C30-2 & 17 & 63.5 & 171 & 36.9 & 37.2 & 36.8 & 3.5 & 3.5 & 3.5 & 26.0 & 26.0 & 27.0 & 1.9 & 2.4 & 4.8 \\
\hline C $30-3$ & 16 & 59.5 & 176 & 37.1 & 37.2 & 37.0 & 2.5 & 3.0 & 3.5 & 20.0 & 24.0 & 25.0 & 3.8 & 3.5 & 2.9 \\
\hline C30-4 & 14 & 63.5 & 171 & 36.8 & 36.7 & 36.7 & 3.5 & 3.5 & 4.0 & 25.0 & 26.0 & 29.0 & 8.0 & 8.1 & 7.4 \\
\hline C30-5 & 12 & 48.5 & 165 & 36.9 & 37.2 & 37.1 & 3.0 & 3.0 & 3.0 & 21.0 & 21.0 & 24.0 & 3.9 & 3.1 & 2.6 \\
\hline Mean & 15.4 & 63.3 & 172.8 & 37.0 & 37.1 & 36.9 & 3.2 & 3.2 & 3.5 & 23.4 & 24.2 & 26.0 & 4.8 & 4.8 & 4.6 \\
\hline ED30-1 & 18 & 71.0 & 183 & 37.2 & 37.1 & 37.1 & 2.0 & 2.5 & 3.0 & 16.0 & 19.0 & 21.0 & 12.6 & 9.2 & 4.4 \\
\hline ED30-2 & 17 & 61.0 & 183 & 36.8 & 37.4 & 36.9 & 2.5 & 2.5 & 3.0 & 20.0 & 18.0 & 22.0 & 5.9 & 3.2 & 5.7 \\
\hline ED30-3 & 16 & 62.0 & 186 & 37.5 & 37.3 & 37.3 & 2.5 & 2.5 & 3.0 & 17.0 & 17.0 & 21.0 & 4.3 & 2.7 & 2.3 \\
\hline ED30-4 & 14 & 59.0 & 169 & 37.0 & 37.1 & 37.1 & 2.5 & 3.0 & 3.0 & 20.0 & 22.0 & 21.0 & 2.9 & 1.8 & 4.7 \\
\hline ED30-5 & 12 & 33.0 & 150 & 37.0 & 37.4 & 36.9 & 3.0 & 3.0 & 3.0 & 22.0 & 21.0 & 22.0 & 4.8 & 4.1 & 2.9 \\
\hline Mean & 15.4 & 57.2 & 174.2 & 37.1 & 37.3 & 37.1 & 2.5 & 2.7 & 3.0 & 19.0 & 19.4 & 21.4 & 6.1 & 4.2 & 4.0 \\
\hline
\end{tabular}

$\varnothing$, without cooling; $v$, wearing a cooling vest; $v+b$, wearing a cooling vest together with a cooling bandana.

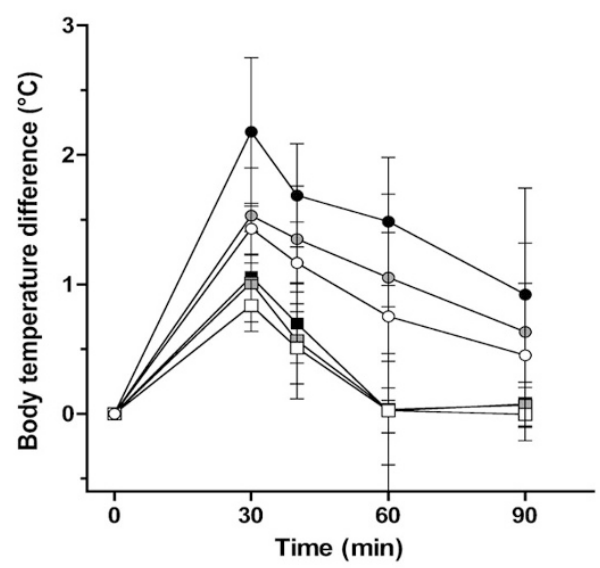

Figure 2. Exercise-induced changes of body core temperature in adolescents at an ambient temperature of $30^{\circ} \mathrm{C}$ with and without cooling devices. Subjects cycled for a maximum of 30 min without cooling (black symbols), wearing a cooling vest (gray symbols) or a cooling vest and a cooling bandana (open symbols). XLHED subjects are displayed as circles and control subjects as squares. Data are displayed as mean $\pm \mathrm{SD}$.

cents disappeared. In comparison with control adolescents wearing both cooling devices, XLHED subjects with cooling vest and cooling bandana cycled for a significantly shorter time period $(p=0.001)$.

\section{DISCUSSION}

This study is the first to demonstrate that XLHED males experience a significantly greater rise in body temperature during and after exercise than healthy controls, which may lead to dangerous heat-related illnesses. It addresses an issue that all families with XLHED-affected individuals know well, but which has not been documented scientifically so far. Infants with XLHED are at increased risk of heat-related brain damage and mortality when compared with healthy infants $(3,9)$. Our findings in older children and adolescents indicate that HED remains a severe disease throughout life, causing substantial health hazards under certain environmental conditions. This underlines the importance of current attempts to develop a novel therapeutic strategy for the treatment of children with XLHED (10-12).

Although controversial (13), thermoregulation in children has been suggested to rely on heat dissipation rather than evaporation and sweating. This may be explained by their greater body surface area $(14,15)$. Here, XLHED children and XLHED adolescents showed an extraordinary increase in body temperature after exercise, indicating an important role of sweating in the thermoregulation of children.

Exercise-induced heat illnesses occur in professional and recreational sports and may range from harmless heat edema, muscular cramps, and orthostatic dysregulation to heat stroke, a life-threatening condition associated with a body core temperature of more than $40^{\circ} \mathrm{C}$ and CNS disturbances $(15,16)$. The extent of exertional hyperthermia that endangers an individual cannot be determined in general. However, it is well known that elevated body temperature leads to diminished endurance and performance in healthy adults and athletes $(5,17,18)$. Discontinuation of exercise when heated may be an important mechanism to prevent catastrophic events such as exertional heat stroke. External cooling is another means of prevention. Although under certain conditions, precooling is known to improve endurance and performance of healthy adults $(5,6,19)$, ice-cooling vests have not been efficient in enhancing endurance, performance, or postexercise cooling in healthy persons $(20,21)$.

Because of the absence or reduced number of sweat glands, HED individuals lack natural evaporative cooling. Therefore, both sports and occupation may be limited solely by heat intolerance (3). To improve their heat tolerance, affected individuals are recommended to make use of artificial evaporative cooling, e.g. by wetting their clothes or sprinkling the skin with water. Similarly, external fleece cooling devices as applied in our study rely on evaporative cooling. The conditions chosen for their evaluation represent typical outdoor environmental conditions at the height of summer. In this setting, XLHED subjects benefited clearly from external evaporative cooling. Although body core temperature could not be normalized, a clinically relevant attenuation of exerciseinduced hyperthermia was observed when XLHED adolescents wore both a cooling vest and a cooling bandana. Arti- 
ficial evaporative cooling reduced the likelihood of potentially endangering exertional hyperthermia. Simpler means of making evaporation available externally such as wetting the clothes or dipping the head into water may also be sufficient to attenuate overheating during exercise. Cold water immersion is an effective cooling modality (7) and proved to be a more efficient means of cooling than ice vests in a group of firefighters performing exercise on a treadmill (22). For children with XLHED, however, a cooling vest or a cooling cap may be a more comfortable way to achieve evaporative cooling or may be more readily accepted than a wet shirt. Therefore, we focused on such cooling devices and did not evaluate other cooling modalities in our study.

In comparison with uncooled control subjects, the trend toward lower performance and shorter self-chosen duration of exercise in XLHED adolescents at $30^{\circ} \mathrm{C}$ was not observed anymore when the latter were equipped with cooling devices, although XLHED subjects wearing both cooling devices cycled significantly shorter than control adolescents with a cooling vest and a cooling bandana. Being equipped with a device in an experimental setting may increase motivation, so that the disappearance of the trend toward lower performance might be explained by a higher motivation to persist in exercising. However, there were no significant differences in endurance when uncooled control subjects were compared with control subjects wearing cooling devices, or when uncooled XLHED males were compared with XLHED males wearing cooling devices.

In summary, this first systematic study of the effects of physical exercise on pediatric HED patients demonstrates a rapid and lasting increase of body temperature in XLHED subjects after cycling in a warm environment, posing them at risk of exercise-induced hyperthermia which may eventually lead to heat-related illnesses. External evaporative skin cooling attenuates exertional overheating in XLHED patients and may enable them to participate in recommendable athletic activities more frequently and, in a number of cases, may help meeting the demands of professional life.

\section{REFERENCES}

1. Itin PH, Fistarol SK 2004 Ectodermal dysplasias. Am J Med Genet 131C:45-51

2. Mikkola ML 2009 Molecular aspects of hypohidrotic ectodermal dysplasia. Am J Med Genet 149A:2031-2036

3. Clarke A, Phillips DI, Brown R, Harper PS 1987 Clinical aspects of X-linked hypohidrotic ectodermal dysplasia. Arch Dis Child 62:989-996

4. Salisbury DM, Stothers JK 1981 Hypohidrotic ectodermal dysplasia and sudden infant death. Lancet 1:153-154

5. Marino FE 2002 Methods, advantages, and limitations of body cooling for exercise performance. Br J Sports Med 36:89-94

6. Duffield R, Marino FE 2007 Effects of pre-cooling procedures on intermittent-sprint exercise performance in warm conditions. Eur J Appl Physiol 100:727-735

7. McDermott BP, Casa DJ, Ganio MS, Lopez RM, Yeargin SW, Armstrong LE, Maresh CM 2009 Acute whole-body cooling for exercise-induced hyperthermia: a systematic review. J Athl Train 44:84-93

8. Darwin C 1885 The Variations of Animals and Plants under Domestication. Vol. 2. 2nd ed. John Murray, London, pp 319-320

9. Blüschke G, Nüsken KD, Schneider H 2010 Prevalence end prevention of severe complications of hypohidrotic ectodermal dysplasia in infancy. Early Hum Dev 86:397-399

10. Mauldin EA, Gaide O, Schneider P, Casal ML 2009 Neonatal treatment with recombinant ectodysplasin prevents respiratory disease in dogs with $\mathrm{X}$-linked ectodermal dysplasia. Am J Med Genet 149A:2045-2049

11. Casal ML, Lewis JR, Mauldin EA, Tardivel A, Ingold K, Favre M, Paradies F, Demotz S, Gaide O, Schneider P 2007 Significant correction of disease after postnatal administration of recombinant ectodysplasin A in canine X-linked ectodermal dysplasia. Am J Hum Genet 81:1050-1056

12. Gaide O, Schneider P 2003 Permanent correction of an inherited ectodermal dysplasia with recombinant EDA. Nat Med 9:614-618

13. Inbar O, Morris N, Epstein Y, Gass G 2004 Comparison of thermoregulatory responses to exercise in dry heat among prepubertal boys, young adults and older males. Exp Physiol 89:691-700

14. Falk B, Dotan R 2008 Children's thermoregulation during exercise in the heat: a revisit. Appl Physiol Nutr Metab 33:420-427

15. Marshall SW 2010 Heat injury in youth sport. Br J Sports Med 44:8-12

16. Howe AS, Boden BP 2007 Heat-related illness in athletes. Am J Sports Med 35:1384-1395

17. González-Alonso J, Teller C, Andersen SL, Jensen FB, Hyldig T, Nielsen B 1999 Influence of body temperature on the development of fatigue during prolonged exercise in the heat. J Appl Physiol 86:1032-1039

18. Nybo L 2008 Hyperthermia and fatigue. J Appl Physiol 104:871-878

19. Ückert S, Joch W 2007 Effects of warm-up and precooling on endurance performance in the heat. Br J Sports Med 41:380-384

20. Brade C, Dawson B, Wallman K, Polglaze T 2010 Postexercise cooling rates in 2 cooling jackets. J Athl Train 45:164-169

21. Duffield R, Dawson B, Bishop D, Fitzsimons M, Lawrence S 2003 Effect of wearing an ice cooling jacket on repeat sprint performance in warm/humid conditions. Br J Sports Med 37:164-169

22. Barr D, Reilly T, Gregson W 2011 The impact of different cooling modalities on the physiological responses in firefighters during strenuous work performed in high environmental temperatures. Eur J Appl Physiol 111:959-967 